\title{
Article \\ Antimicrobial Activity of the Peptide LfcinB15 against Candida albicans
}

\author{
Che-Kang Chang ${ }^{1}$, Mou-Chieh Kao ${ }^{2,3, *(D)}$ and Chung-Yu Lan $1,3, *$ (D) \\ 1 Institute of Molecular and Cellular Biology, National Tsing Hua University, Hsinchu 30013, Taiwan; \\ steve2202543@gapp.nthu.edu.tw \\ 2 Institute of Molecular Medicine, National Tsing Hua University, Hsinchu 30013, Taiwan \\ 3 Department of Life Science, National Tsing Hua University, Hsinchu 30013, Taiwan \\ * Correspondence: mckao@life.nthu.edu.tw (M.-C.K.); cylan@life.nthu.edu.tw; (C.-Y.L.); \\ Tel.: +886-3-5742473 (M.-C.K.); +886-3-5742472 (C.-Y.L.)
}

check for

updates

Citation: Chang, C.-K.; Kao, M.-C.; Lan, C.-Y. Antimicrobial Activity of the Peptide LfcinB15 against Candida albicans. J. Fungi 2021, 7, 519.

https://doi.org/10.3390/jof7070519

Academic Editor: Richard Calderone

Received: 17 May 2021

Accepted: 25 June 2021

Published: 29 June 2021

Publisher's Note: MDPI stays neutral with regard to jurisdictional claims in published maps and institutional affiliations.

Copyright: (c) 2021 by the authors. Licensee MDPI, Basel, Switzerland. This article is an open access article distributed under the terms and conditions of the Creative Commons Attribution (CC BY) license (https:// creativecommons.org/licenses/by/ $4.0 /)$.

\begin{abstract}
Lactoferricin (Lfcin) is an amphipathic, cationic peptide derived from proteolytic cleavage of the N-lobe of lactoferrin (Lf). Lfcin and its derivatives possess broad-spectrum antibacterial and antifungal activities. However, unlike their antibacterial functions, the modes of action of Lfcin and its derivatives against pathogenic fungi are less well understood. In this study, the mechanisms of LfcinB15, a derivative of bovine Lfcin, against Candida albicans were, therefore, extensively investigated. LfcinB15 exhibited inhibitory activity against planktonic cells, biofilm cells, and clinical isolates of C. albicans and non-albicans Candida species. We further demonstrated that LfcinB15 is localized on the cell surface and vacuoles of C. albicans cells. Moreover, LfcinB15 uses several different methods to kill C. albicans, including disturbing the cell membrane, inducing reactive oxygen species (ROS) generation, and causing mitochondrial dysfunction. Finally, the Hog1 and $\mathrm{Mkc1}$ mitogen-activated protein kinases were both activated in C. albicans cells in response to LfcinB15. These findings help us to obtain more insight into the complex mechanisms used by LfcinB15 and other Lfcin-derived peptides to fight fungal pathogens.
\end{abstract}

Keywords: C. albicans; antimicrobial peptide; LfcinB15

\section{Introduction}

Candida albicans is a commensal yeast inhabiting the skin and various mucosal surfaces of the human body. However, C. albicans is also an opportunistic pathogen, particularly for immunocompromised patients, and can cause a range of diseases such as superficial infections and invasive candidiasis, with high mortality rates [1-3]. Moreover, the emergence of antifungal resistance in C. albicans has become a serious threat in the clinical setting [4].

Antimicrobial peptides (AMPs) are important components of the human innate immune system and are considered promising candidates for developing new antifungals for the treatment of drug-resistant infections [5,6]. AMPs generally consist of 10 to 100 amino acid residues and are divided into four classes based on their secondary structures, including $\alpha$-helical, $\beta$-sheet, extended conformation and $\beta$-hairpin or loop peptides $[7,8]$. Moreover, antimicrobial activities are diverse among different types of AMPs. Some AMPs interact directly with the negatively charged cytoplasmic membrane of microbes, leading to disruption of the membrane [9]. Alternatively, some AMPs translocate into the cytosol to interfere with distinct bioprocesses such as DNA replication and protein synthesis [10]. For example, Tur1A and histatin 5 (Hst 5) translocate intracellularly and exert their antimicrobial activity without membrane disruption [11,12].

Lactoferrin (Lf) is a glycoprotein belonging to the transferrin family of proteins, which are able to bind and transfer iron(III) ions [13]. Human Lf consists of two homologous $\mathrm{N}$ - and C-terminal globular lobes [14] and is found in specific granules of neutrophils, epithelia and body secretions such as milk and saliva [14]. Importantly, human Lf possesses 
antimicrobial, antioxidant, antitumor, antiallergic, anti-inflammatory and immunoregulatory activities, and iron sequestration is associated with its ability to inhibit bacterial growth [15]. In addition to human Lf, bovine Lf is another well-studied form of lactoferrin, sharing high sequence homology with human Lf [14]. Moreover, lactoferricin B (LfcinB) is a 25-amino-acid peptide corresponding to residues 17-41 of bovine Lf generated by acid-pepsin digestion. LfcinB not only has antiviral, antitumor and immunological properties [16] but also notably exhibits even more potent antibacterial activity than intact bovine and human Lf [17-20]. In addition, LfcinB has great efficacy against a broad spectrum of fungi, including species of Candida, Cryptococcus, Aspergillus and Penicillium [21].

Many derivatives of bovine LfcinB have been generated and characterized. For example, the peptide consisting of the 1-10, 4-12 or 2-21 residues of LfcinB (corresponding to positions $17-26,20-28$ or 18-37 of bovine Lf, respectively), exhibiting potent candidacidal activity [21]. Moreover, LfcinB15 is a 15-residue truncated fragment corresponding to residues 1-15 of LfcinB (i.e., positions 17-31 of bovine Lf) and still maintains nearly the same effective antibacterial activity as its parental LfcinB [22]. In addition, LfcinB15 showed low hemolytic activity (less than $8 \%$ lysis) with the concentration ranging from 4 to $256 \mu \mathrm{g} / \mathrm{mL}$ [22]. However, the antifungal activity and the detailed fungal killing mechanism of LfcinB15 have not yet been characterized.

In this study, we investigated LfcinB15 to gain a better understanding of the activity and modes of action of LfcinB-derived peptides against C. albicans. First, LfcinB15 exhibited antifungal activity against planktonic cells, biofilm cells and clinical isolates of C. albicans and non-albicans Candida species. Second, LfcinB15 exerted anti-C. albicans activity through multiple modes of action, including membrane disruption, ROS accumulation and mitochondrial dysfunction. Finally, the $C$. albicans stress response protein kinases Hog1 and Mkc1 were activated by LfcinB15, and the HOG1-deleted (hog1 $\Delta$ ) mutant was significantly resistant to LfcinB15. Together, these findings provide insights into the complex mechanisms of LfcinB15 and LfcinB-derived peptides against fungal pathogens.

\section{Materials and Methods}

\subsection{C. albicans Strains, Media and Growth Conditions}

The C. albicans SC5314 strain was used throughout this study unless stated otherwise. The C. albicans mutant strains that are deficient in mitochondrial electron transport chain (ETC) and their parental strain SN250 [23] were used as indicated. For the determination of minimal inhibitory concentration (MIC), different C. albicans and non-albicans Candida clinical isolates were also used [24]. Cells were maintained at $-80^{\circ} \mathrm{C}$ and plated on YPD agar ( $1 \%$ yeast extract, $2 \%$ peptone, $2 \%$ glucose and $1.5 \%$ agar) before each experiment. A single colony was inoculated into YPD broth and grown at $30^{\circ} \mathrm{C}$ overnight $(\sim 16 \mathrm{~h})$ with shaking at $180 \mathrm{rpm}$. The overnight culture was subcultured in YPD (with an initial concentration $\sim 3 \times 10^{6} \mathrm{CFU} / \mathrm{mL}$ ) and further grown at $30^{\circ} \mathrm{C}$ to the exponential phase.

\subsection{Peptides and Reagents}

LfcinB15 (FKCRRWQWRMKKLGA-NH ${ }_{2}$ ) and fluorescein isothiocyanate (FITC)-conjugated LfcinB15 (FITC-FKCRRWQWRMKKLGA-NH $\mathrm{N}_{2}$ ) were synthesized by Mission Biotech, Inc. (Taipei, Taiwan). The purity of the peptides was determined by reversed-phase highperformance liquid chromatography (HPLC, Shimadzu, Kyoto, Japan) and mass spectrometry to be $>95 \%$ pure. The stock solution $(5 \mathrm{mg} / \mathrm{mL})$ was prepared by dissolving LfcinB15 or FITC-LfcinB15 in sterile double-distilled water. All reagents were purchased from Sigma-Aldrich (St. Louis, MO, USA) unless stated otherwise.

\subsection{Cell Susceptibility to LfcinB15}

Cell susceptibility to LfcinB15 was examined by colony forming unit (CFU) counting and propidium iodide (PI) staining. Briefly, C. albicans cells were suspended to a final concentration of $\sim 3 \times 10^{8} \mathrm{CFU} / \mathrm{mL}$ with $12.5 \mathrm{mM}$ sodium acetate in a $1.5 \mathrm{~mL}$ microtube and $100 \mu \mathrm{L}$ of $C$. albicans cell suspension was incubated with or without different concentrations 
of LfcinB15 at $37^{\circ} \mathrm{C}$ for $1 \mathrm{~h}$. Subsequently, cells were 10 -fold serially diluted, and grown on YPD agar plates and incubated at $30^{\circ} \mathrm{C}$ overnight, and the number of CFUs was counted.

For PI staining, cells $\left(\sim 3 \times 10^{8} \mathrm{CFU} / \mathrm{mL}\right.$ in $12.5 \mathrm{mM}$ sodium acetate $)$ were treated with or without different concentrations of LfcinB15 at $37^{\circ} \mathrm{C}$ for $1 \mathrm{~h}$, harvested by centrifugation, washed with phosphate buffered saline (PBS), resuspended in a PI staining solution $\left(6 \mu \mathrm{g} / \mathrm{mL}\right.$ PI in PBS), and incubated at $37^{\circ} \mathrm{C}$ for $5 \mathrm{~min}$. PI-positive cells were measured using an Accuri C6 flow cytometer (BD Biosciences, San Jose, CA, USA), and data were processed using BD Accuri C6 software.

\subsection{Minimal Inhibitory Concentration (MIC) of LfcinB15}

The MIC of clinically isolated Candida strains to LfcinB15 was determined by the broth dilution method as described in the Clinical and Laboratory Standards Institute document M27-A3 [25], with some modifications, as previously described [26]. Briefly, cells were diluted to a final concentration of $2 \times 10^{4} \mathrm{CFU} / \mathrm{mL}$ with low salt LYM broth [26]. Two hundred microliters of cell suspension were added to each well of a 96-well microplate and incubated with the indicated concentration of LfcinB15 at $37^{\circ} \mathrm{C}$ for $48 \mathrm{~h}$. The absorbance at $595 \mathrm{~nm}$ was then measured using a Bio-Rad iMarkTM microplate reader (Bio-Rad, Hercules, CA, USA). The MIC was defined as the concentration of LfcinB15 that caused a complete inhibition in growth relative to the LfcinB15-free control. The experiments were repeated at least three times.

\subsection{Assessment of Biofilm Formation}

The XTT reduction assay was performed to measure biofilm formation, as previously described [27]. After overnight growth, $C$. albicans cells were suspended to a final concentration of $3 \times 10^{5} \mathrm{CFU} / \mathrm{mL}$ in RPMI 1640 medium (Thermo Fisher, Waltham, MA, USA) with $3.453 \%$ MOPS and $1.8 \%$ glucose. Cells were then added to each well of a 96-well polystyrene microplate and incubated for $24 \mathrm{~h}$ at $37^{\circ} \mathrm{C}$ with $5 \% \mathrm{CO}_{2}$. Subsequently, the adherent cells in the biofilm were washed and incubated with or without different concentrations of LfcinB15 (in $12.5 \mathrm{mM}$ sodium acetate) at $37^{\circ} \mathrm{C}$ for $1 \mathrm{~h}$. The supernatant in each well was carefully removed, and $300 \mu \mathrm{L}$ of 2,3-bis-(2-methoxy-4-nitro-5-sulfophenyl)-2Htetrazolium-5-carboxanilide (XTT) with $0.5 \mu \mathrm{M}$ menadione in PBS was added to the biofilm cells and incubated at $37^{\circ} \mathrm{C}$ for $15 \mathrm{~min}$. The XTT reduction was measured at $490 \mathrm{~nm}$ using a Bio-RAD iMarkTM microplate reader.

For the examination of the $C$. albicans biofilm structure, biofilms were formed on Thermanox polystyrene coverslips (Thermo Fisher) and examined by SEM, as previously described [27]. Briefly, biofilm cells on coverslips were treated with or without LfcinB15 (100 and $200 \mu \mathrm{g} / \mathrm{mL}$ ), incubated at $37^{\circ} \mathrm{C}$ for $1 \mathrm{~h}$, and washed twice with PBS. The coverslips were then fixed with $3.75 \%$ formaldehyde (in PBS) at room temperature (RT) for $40 \mathrm{~min}$, followed by incubation with 1\% osmium tetroxide (in PBS; Electron Microscopy Sciences, Hatfield, PA, USA) for $5 \mathrm{~min}$ and dehydration with serial ethanol solutions [27]. The samples were dried in a $60^{\circ} \mathrm{C}$ oven. Finally, the biofilms were examined, and micrographs were collected using SEM (Hitachi, S-4700, Type II).

\subsection{Fluorescence Microscopy}

To determine the subcellular localization of LfcinB15, cells were simultaneously stained with FITC-LfcinB15 and calcofluor white (CFW), 7-amino-4-chloromethylcoumarin (CellTracker Blue CMAC, PerkinElmer, Carlsbad, CA, USA), MitoTracker red (MTR; Invitrogen, Eugene, OR, USA) or 4',6-diamidino-2-phenylindole (DAPI). Briefly, C. albicans cells were suspended to a final concentration of $3 \times 10^{8} \mathrm{CFU} / \mathrm{mL}$ with $12.5 \mathrm{mM}$ sodium acetate and incubated with $6.25 \mu \mathrm{g} / \mathrm{mL}$ LfcinB 15 at $37^{\circ} \mathrm{C}$ for $15 \mathrm{~min}$. For organelle or cell wall staining, cells were independently incubated with $1 \mu \mathrm{g} / \mathrm{mL} \mathrm{CFW}, 100 \mu \mathrm{M}$ CMAC, $75 \mathrm{nM}$ MTR, or $1 \mu \mathrm{g} / \mathrm{mL}$ DAPI. The samples were subsequently examined using a CLSM system (Zeiss LSM800 with Airyscan; Carl Zeiss, Jena, Germany) at $100 \times$ magnification. The images were processed using ZEISS ZEN software version 1.1.2.0. 


\subsection{Liposome Preparation and Determination of Membrane Disturbance by Measuring Calcein Leakage}

Small unilamellar liposome vesicles (SUVs; $100 \mathrm{~nm}$ diameter) were prepared, as previously described [28], with some modifications. Briefly, phosphatidylcholine (PC), phosphatidylethanolamine (PE) and phosphatidylinositol were mixed with ergosterol at a ratio of 5:4:1:2 $(w / w / w / w)$ in chloroform. PC, PE and phosphatidylinositol were purchased from Avanti Polar Lipids (Alabaster, AL, USA). The mixture was then dried using a stream of nitrogen gas, and the lipid film was vigorously mixed with liposome buffer (70 $\mathrm{mM}$ calcein, $10 \mathrm{mM}$ Tris, $75 \mathrm{mM} \mathrm{NaCl}$, and 0.1 mM EDTA, pH 7.4) for $30 \mathrm{~min}$. Subsequently, the solution was repeatedly frozen and thawed with a $-80^{\circ} \mathrm{C}$ refrigerator for 10 cycles and extruded through a $100 \mathrm{~nm}$ polycarbonate filter 5 times. The SUVs loaded with calcein were purified with a Sephadex G50 gel filtration column.

To detect the release of calcein, freshly prepared SUVs were treated with or without various concentrations of LfcinB15 for $5 \mathrm{~min}$ at RT in a reaction buffer $(10 \mathrm{mM}$ Tris, $150 \mathrm{mM}$ $\mathrm{NaCl}$, and $0.1 \mathrm{mM}$ EDTA, $\mathrm{pH} 7.4$ ). SUVs treated with $0.1 \%$ Triton $\mathrm{X}-100$ were used as a positive control. The fluorescence of calcein release was measured using a Victor $3^{\mathrm{TM}}$ Plate Reader (PerkinElmer, Santa Clara, CA, USA) at excitation and emission wavelengths of 495 and $515 \mathrm{~nm}$, respectively. The percentage of calcein release was calculated as follows: calcein release $=\left(\mathrm{F}-\mathrm{F}_{0}\right) /\left(\mathrm{F}_{\mathrm{t}}-\mathrm{F}_{0}\right) \times 100 \%$, where $\mathrm{F}$ is the fluorescence intensity after LfcinB15 treatment, $F_{t}$ is the fluorescence intensity with Triton X-100 treatment, and $F_{0}$ is the fluorescence intensity of the untreated control SUVs.

\subsection{Measurement of Intracellular ROS}

Intracellular ROS levels were measured using dihydroethidium (DHE), 2', $7^{\prime}$ dichlorodihydrofluorescein diacetate $\left(\mathrm{H}_{2} \mathrm{DCFDA}\right)$, and MitoSOX red (Invitrogen), as previously described [29]. Briefly, cells $\left(3 \times 10^{8} \mathrm{CFU} / \mathrm{mL}\right)$ were treated with or without different concentrations of LfcinB15 for $1 \mathrm{~h}$. The cells were collected, washed with PBS, resuspended in PBS containing either $20 \mu \mathrm{g} / \mathrm{mL} \mathrm{H}_{2}$ DCFDA or $5 \mu \mathrm{M}$ MitoSOX Red, and incubated at $30{ }^{\circ} \mathrm{C}$ for 30 and $20 \mathrm{~min}$, respectively. For DHE staining, cells were resuspended in PBS containing $20 \mu \mathrm{M} \mathrm{DHE}$ and incubated at $37^{\circ} \mathrm{C}$ for $5 \mathrm{~min}$. The fluorescence intensity was measured using an AccuriTM C6 flow cytometer (BD Biosciences), and the mean fluorescence intensity was calculated using BD Accuri C6 software.

\subsection{Cell Rescue Assay Using ROS Scavengers}

To determine the relationship between ROS generation and $C$. albicans killing by LfcinB15, the ROS scavengers NAC and glutathione were used. Briefly, $3 \times 10^{8} \mathrm{CFU} / \mathrm{mL}$ cells were suspended in $12.5 \mathrm{mM}$ sodium acetate and incubated at $37{ }^{\circ} \mathrm{C}$ with either $60 \mathrm{mM}$ N-acetyl cysteine (NAC) or $15 \mathrm{mM}$ glutathione for $30 \mathrm{~min}$, respectively. Cells were harvested, washed with $12.5 \mathrm{mM}$ sodium acetate, treated with or without different concentrations of LfcinB15, and incubated at $37^{\circ} \mathrm{C}$ for $1 \mathrm{~h}$. Cells were then plated on YPD agar plates and incubated overnight at $30^{\circ} \mathrm{C}$, and the number of CFUs from cells treated with LfcinB15 was counted and normalized to that of the untreated control. The results were reported as percentages.

\subsection{Protein Extraction and Western Blotting}

Protein extraction and western blotting were performed as previously described [30]. An anti-phospho-p38 (Thr180/Tyr182) monoclonal antibody (\#9211, Cell Signaling Technology, Danvers, MA, USA) and Hog1 (y-215) antibody (sc-9079, Santa Cruz Biotechnology, Inc., Dallas, TX, USA) were used to detect phospho-Hog1 and total Hog1, respectively. Moreover, an anti-p-P44/42 MAPK antibody (4270S, Cell Signaling Technology) was used for the detection of both phosphor-Mkc1 and phosphor-Cek1, and a GAPDH antibody (GTX100118, GeneTex, Hsinchu, Taiwan) was used for GAPDH. Horseradish peroxidase (HRP)-conjugated goat anti-rabbit IgG (GTX213110, GeneTex) was used as the secondary antibody. The blot was visualized using the Western Lightning Plus-ECL Enhanced Chemi- 
luminescence Substrate kit (PerkinElmer) and an ImageQuant LAS 4000 Biomolecular Imager (GE Healthcare Life Science, Marlborough, MA, USA).

\subsection{Measurement of the Mitochondrial Membrane Potential}

The mitochondrial membrane potential was measured by staining cells with rhodamine 123, as previously described [31], and JC-1, according to the manufacturer's instructions (Thermo Fisher) but with some modifications. For rhodamine 123 staining, cells $\left(3 \times 10^{8} \mathrm{CFU} / \mathrm{mL}\right)$ were treated with or without different concentrations of LfcinB15 for $1 \mathrm{~h}$, harvested by centrifugation, resuspended with $25 \mu \mathrm{M}$ rhodamine 123 (in $50 \mathrm{mM}$ sodium citrate) and incubated at $30^{\circ} \mathrm{C}$ for $10 \mathrm{~min}$. For JC-1 staining, cells were harvested, resuspended with $2 \mu \mathrm{M} \mathrm{JC}-1$ (in PBS) and incubated at $37^{\circ} \mathrm{C}$ for $15 \mathrm{~min}$. After staining, cells were washed three times with PBS, and the fluorescence intensity was measured using an AccuriTM C6 flow cytometer (BD Biosciences). Cells without AMP treatment were used as negative controls, whereas cells treated with $15 \mathrm{mM}$ hydrogen peroxide $\left(\mathrm{H}_{2} \mathrm{O}_{2}\right)$ for $1 \mathrm{~h}$ were used as positive controls.

\subsection{Measurement of Intracellular and Extracellular ATP Levels}

The ATP level was determined with a bioluminescence assay using an ATP Determination Kit (A22066, Invitrogen). Briefly, C. albicans cells were diluted to a final concentration of $3 \times 10^{8} \mathrm{CFU} / \mathrm{mL}$ with $12.5 \mathrm{mM}$ sodium acetate, incubated with or without different concentrations of LfcinB 15 at $37^{\circ} \mathrm{C}$ for $1 \mathrm{~h}$, and collected by centrifugation. The supernatant was kept on ice before use for measuring the extracellular ATP level, while the cell pellets were broken, as previously described [30], for measuring intracellular ATP.

For ATP measurement, the ATP Determination Kit was used according to the manufacturer's instructions (Invitrogen). Briefly, $1 \times$ reaction buffer containing $0.5 \mathrm{mM}$ D-luciferin, $1.25 \mu \mathrm{g} / \mathrm{mL}$ firefly luciferase, and $1 \mathrm{mM}$ DTT was freshly prepared before each experiment, and $10 \mu \mathrm{L}$ of the supernatant or cell lysate was mixed with $90 \mu \mathrm{L}$ of the reaction buffer. Then, the mixture was placed into each well of a black 96-well microplate, and luminescence was read using a Victor3 ${ }^{\text {TM }}$ Plate Reader (PerkinElmer) at $560 \mathrm{~nm}$. A standard curve of increasing ATP concentrations $(0,0.01,0.1,1 \mu \mathrm{M})$ was created. Signals represented at least three independent experiments were obtained and the ATP concentration of each sample was calculated from the standard curve.

\subsection{Oxygen Consumption Rate (OCR) Measurement Using High-Resolution Respirometry}

The OCR was measured using an Oxygraph-O2k system (Oroboros Instruments, Innsbruck, Austria). Briefly, C. albicans cells were diluted to a final concentration of $3 \times 10^{8} \mathrm{CFU} / \mathrm{mL}$ with $12.5 \mathrm{mM}$ sodium acetate and incubated, with or without $12.5 \mu \mathrm{g} / \mathrm{mL}$ LfcinB15, at $37^{\circ} \mathrm{C}$ for $1 \mathrm{~h}$. After incubation, cells were diluted again with PBS to a final concentration of $6 \times 10^{6} \mathrm{CFU} / \mathrm{mL}$. The Oroboros Oxygraph-O2k system was first air calibrated with PBS following the manufacturer's instructions. Then, $3 \mathrm{~mL}$ of each sample was loaded into the $\mathrm{O} 2 \mathrm{k}$ chambers, and the $\mathrm{OCR}$ was monitored until the signal reached a plateau. For CCCP titration, cells were slowly titrated with $1.5 \mu \mathrm{L}$ of stock CCCP solution (10 mM in dimethyl sulfoxide or DMSO) 10-20 times until the OCR stopped increasing. Subsequently, cells from each sample were grown on YPD agar plates and incubated at $30^{\circ} \mathrm{C}$ overnight, and the number of CFUs was counted. The OCR was calculated as follows: OCR per million CFUs = average OCR reading in the plateau $(\mathrm{pmol} / \mathrm{s} \times \mathrm{mL}) /$ the number of millions of CFUs, where $\mathrm{s}$ is seconds.

\subsection{Determination of the Association between LfcinB15 and Mitochondrial Respiration}

Susceptibility to rotenone, sodium azide $\left(\mathrm{NaN}_{3}\right)$ and carbonyl cyanide mchlorophenylhydrazone (CCCP) was assessed to correlate LfcinB15 killing with mitochondrial respiration. For the experiments with rotenone treatment, cells $\left(3 \times 10^{8} \mathrm{CFU} / \mathrm{mL}\right)$ were suspended in $12.5 \mathrm{mM}$ sodium acetate, treated with $0.31 \mathrm{mM}$ rotenone, and incubated at $37^{\circ} \mathrm{C}$ for $30 \mathrm{~min}$. Cells were harvested, washed with $12.5 \mathrm{mM}$ sodium acetate, and 
incubated with or without different concentrations of LfcinB15 at $37^{\circ} \mathrm{C}$ for $1 \mathrm{~h}$. For the treatment with $\mathrm{NaN}_{3}$, cells were harvested, washed with $12.5 \mathrm{mM}$ sodium acetate, and incubated with $5 \mathrm{mM} \mathrm{NaN}_{3}$ at $37^{\circ} \mathrm{C}$ for $10 \mathrm{~min}$. The $\mathrm{NaN}_{3}$-treated cells were further treated with or without different concentrations of LfcinB15 and incubated at $37^{\circ} \mathrm{C}$ for $1 \mathrm{~h}$. For the treatment with CCCP, cells were coincubated with or without different concentrations of LfcinB15 and $50 \mu \mathrm{M} \mathrm{CCCP}$ at $37^{\circ} \mathrm{C}$ for $1 \mathrm{~h}$. In all the experiments, cells were then plated on YPD agar plates and incubated overnight at $30^{\circ} \mathrm{C}$. The number of CFUs was counted and normalized to that of the control cells. The results are reported as percentages.

The susceptibility of the C. albicans mutants that are deficient in mitochondrial ETC (orf19.4758, orf19.7590, mci4, orf19.1710, and cox4) and ypt72 genes to LfcinB15 were assessed by CFU counting as described above.

\subsection{Statistical Analysis}

The two-tailed Student's $t$-test was applied to assess significant differences between samples. Statistical significance was indicated with a $p$-value $<0.05$.

\section{Results}

\subsection{LfcinB15 Exerts Anti-Candida Activity against Both Planktonic and Biofilm Cells}

To determine the ability of LfcinB15 to kill C. albicans, CFU counting and PI staining were performed. The viability of cells treated with LfcinB15 was compared to that of untreated control cells. The results indicated that cell viability was decreased as the LfcinB15 concentration increased (Figure 1a). Cell viability was $\sim 80 \%$ and $\sim 40 \%$ after treatment with $12.5 \mu \mathrm{g} / \mathrm{mL}$ and $25 \mu \mathrm{g} / \mathrm{mL}$ LfcinB15, respectively. Moreover, PI is a cell-impermeable intercalating dye that stains nucleic acids of dead cells and is used to distinguish between living and dead cells. Cell death (PI-positive cells) was induced with increasing concentrations of LfcinB15 (Figure 1b).

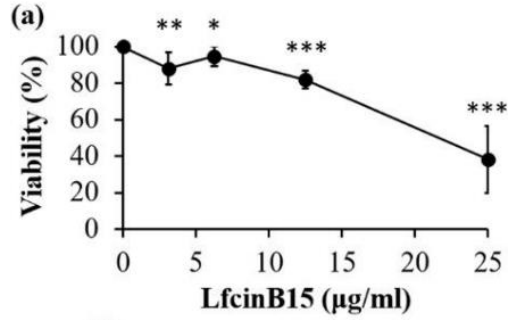

(d)

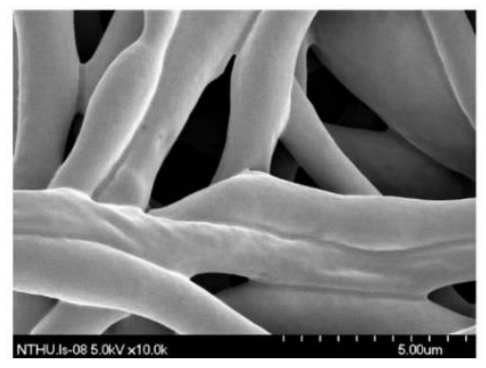

(b)
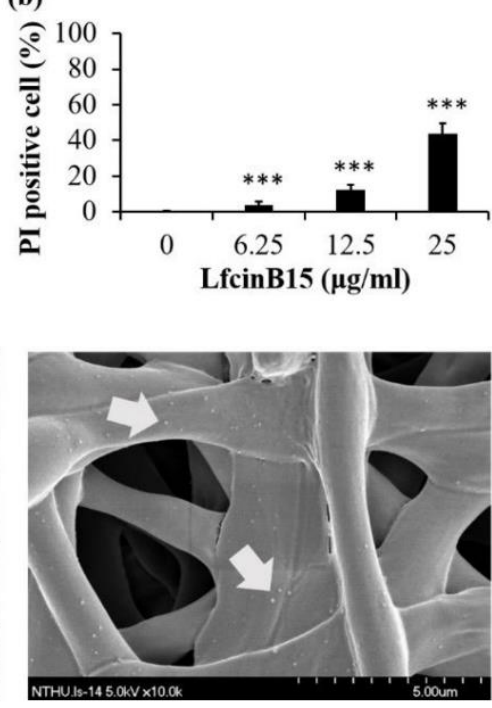

(c)
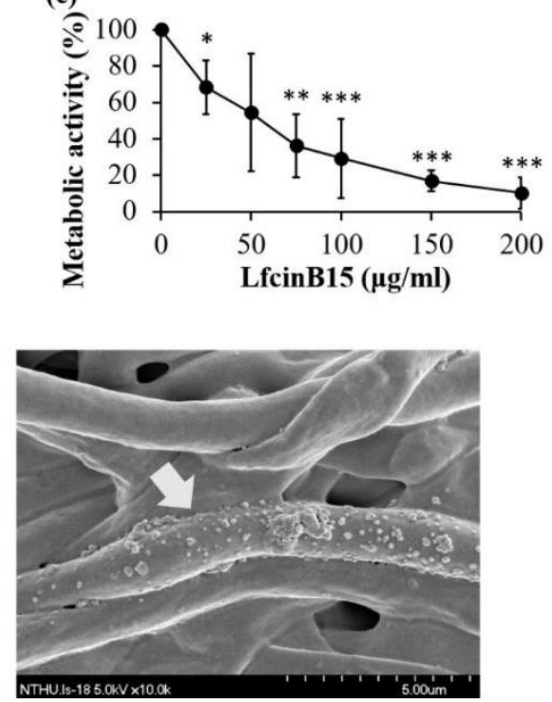

Figure 1. LfcinB15 has anti-Candida activity. (a) Killing of C. albicans by LfcinB15 was determined by counting the number of CFUs and expressed as the percentage of viable cells. The results are presented as the mean \pm standard deviation (SD) of three independent experiments; ${ }^{*} p<0.05 ;{ }^{* *} p<0.01 ;{ }^{* * *} p<0.001$. (b) Killing of C. albicans by LfcinB15 was detected by PI staining, and the dead (PI-positive) cells were quantified by flow cytometry and reported as a percentage. The results are presented as the mean $\pm \mathrm{SD}$ of three independent experiments; ${ }^{* *} p<0.001$. (c) Reduction in metabolic activity of C. albicans biofilms after LfcinB15 treatment. The results are reported as percentages, and error bars represent the SD from the mean of three independent experiments. ${ }^{*} p<0.05 ;{ }^{* *} p<0.01 ;{ }^{* *} p<0.001$. (d) The morphology of biofilm cells treated without (left) and with $100 \mu \mathrm{g} / \mathrm{mL}$ (middle) and $200 \mu \mathrm{g} / \mathrm{mL}$ (right) LfcinB15 was examined by SEM at a magnification of $\times 10,000$. Arrows indicate the rough appearance of protuberances. 
Biofilms are important for C. albicans survival and dispersion for colonization of new niches within the human host. Moreover, biofilm cells exhibit resistance to currently available antifungals and are closely related to infection [32]. To assess the efficacy of LfcinB15 against biofilm cells, biofilms were formed and incubated with different concentrations of LfcinB15, followed by measuring the metabolic activity of $C$. albicans biofilms using the $X T T$ reduction assay [27]. The results indicated that increasing concentrations of LfcinB15 significantly reduced biofilm cells compared to the control without LfcinB15 treatment (Figure 1c). To further examine the effect of LfcinB15 on biofilm cells, the structure of the formed biofilms was also examined by scanning electron microscopy (SEM). As indicated, the surfaces of cells without LfcinB15 treatment were smooth, whereas the surfaces of treated cells showed a rough appearance with protuberances (Figure 1d). Overall, LfcinB15 was effective against both planktonic and biofilm C. albicans cells in a dose-dependent manner, and the structure of the biofilm was altered by LfcinB15.

\subsection{LfcinB15 Also Exerts Antifungal Activity against Clinical Isolates of Candida Species}

To further verify the efficacy of LfcinB15, the susceptibility of fourteen C. albicans and non-albicans Candida clinical isolates to the peptide was determined by the broth dilution method [25]. The results showed that the minimal inhibitory concentration (MIC) of LfcinB15 in ten of the clinical isolates was similar to or lower than that in the reference C. albicans SC5314 strain (Table 1). Notably, among these ten isolates, YH050072, YH050075, YLO86, YH050007, YH050013, and YH050114 are fluconazole resistant [24]. Moreover, C. glabrata isolates were much more resistant to LfcinB15 than C. albicans and other nonalbicans Candida isolates. This finding is consistent with previous studies in which clinical isolates of $C$. glabrata were found to be somehow more resistant than other Candida species to different AMPs [33-35].

Table 1. Antifungal activity of LfcinB15 against clinical isolates of Candida spp.

\begin{tabular}{cccc}
\hline Candida spp. & Source $^{\text {a }}$ & Strains & MIC $(\mu \mathbf{g} / \mathbf{m L})$ \\
\hline C. albicans & ATCC MYA-2876 & SC5314 & 6.25 \\
C. albicans & ATCC90028 & YLO12 & 6.25 \\
C. albicans & HIV patient & YH050001 & 6.25 \\
C. albicans & HIV patient & YH050005 & 12.5 \\
C. albicans & HIV patient & YH050072 & 6.25 \\
C. glabrata & ATCC9003 & YLO8 & $>50$ \\
C. glabrata & HIV patient & YH050105 & 50 \\
C. krusei & ATCC6258 & YLO6 & 12.5 \\
C. krusei & HIV patient & YH050075 b & 6.25 \\
C. tropicalis & ATCC13803 & YLO86 ${ }^{\mathrm{b}}$ & 3.125 \\
C. tropicalis & HIV patient & YH050007 b & 3.125 \\
C. tropicalis & HIV patient & YH050013 b & 6.25 \\
C. tropicalis & HIV patient & YH050114 b & 6.25 \\
C. parapsilosis & ATCC22019 & YLO7 & 3.125 \\
C. dubliniensis & HIV patient & YH050092 & 12.5 \\
\hline
\end{tabular}

a HIV patient, Candida strains isolated from HIV-infected patients. ${ }^{\mathrm{b}}$ Fluconazole-resistant isolates.

\subsection{LfcinB15 Disturbs Membrane Integrity and Enters C. albicans Cells}

To reveal the mechanisms of action of LfcinB15, FITC-conjugated LfcinB15 was used to determine the cellular localization of the peptide. C. albicans was coincubated with FITCLfcinB15 and CFW, a fluorescent cell wall stain, followed by examining cells with confocal laser scanning microscopy (CLSM). Figure 2 shows that FITC-LfcinB15 colocalized with $\mathrm{CFW}$, suggesting that the peptide interacts with the cell surface of $C$. albicans. Moreover, to determine whether the peptide can further gain access into cells, C. albicans cells were coincubated with FITC-LfcinB15 and various organelle-specific dyes, including DAPI, CMAC and MTR, which are used for staining the nucleus, vacuole and mitochondria, respectively. The images indicated that FITC-LfcinB15 colocalized with CMAC but not 
DAPI or MTR (Figure 2). Therefore, the peptide is not only associated with the cell surface but can also enter the cell to accumulate in vacuoles.

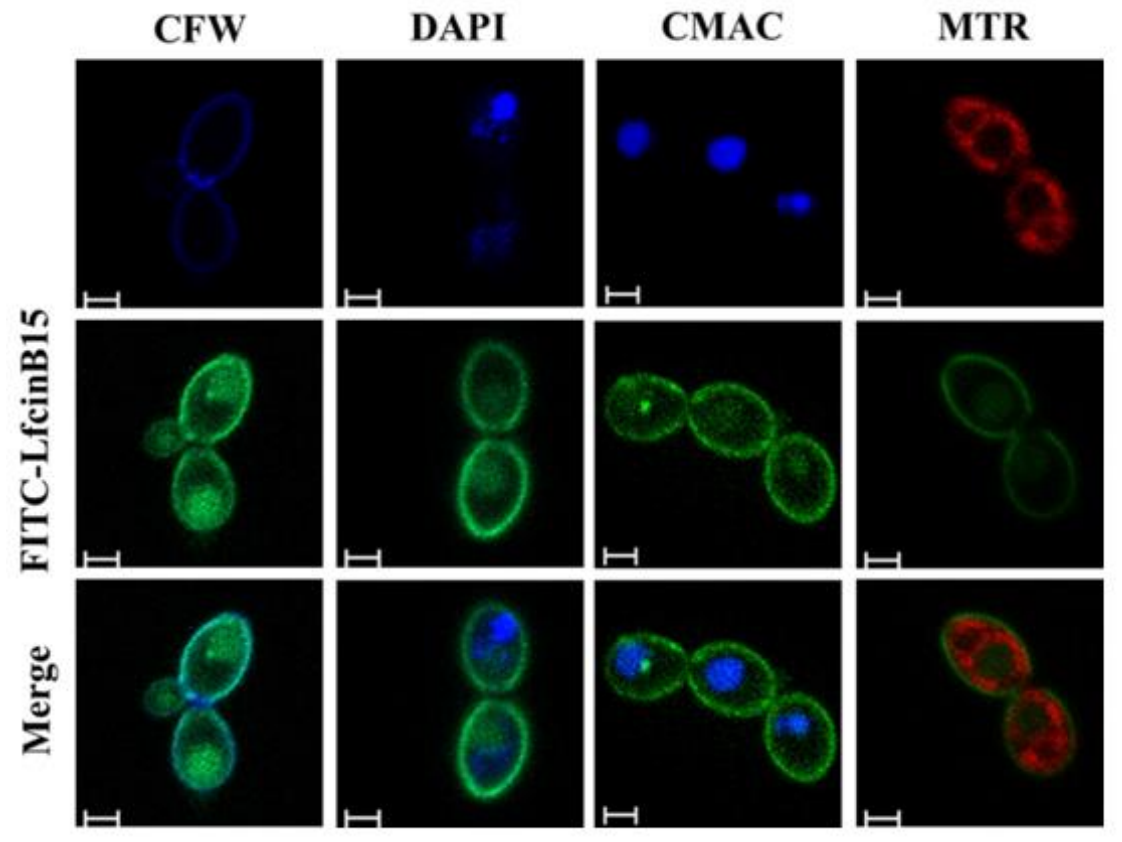

Figure 2. FITC-labeled LfcinB15 is mainly localized to the C. albicans cell surface and vacuole. Fluorescence images show different subcellular localization of FITC-LfcinB15 by CLSM at a magnification of $\times 100$. CFW: calcofluor white; DAPI: 4',6-diamidino-2-phenylindole; CMAC: CellTracker Blue CMAC (7-amino-4-chloromethylcoumarin) dye; MTR: MitoTracker red. Scar bar: $2 \mu \mathrm{m}$.

AMPs generally function in either membrane or nonmembrane targeting [36]. Because LfcinB15 associates with the cell surface (Figure 2), we were thus interested in further determining whether LfcinB15 has membrane-disturbing ability, which was assessed by a SUV-calcein leakage assay. These SUVs mimicking the plasma membrane of $C$. albicans were prepared as previously described [28]. In this assay, calcein-loaded SUVs were treated with various concentrations of LfcinB15 for $5 \mathrm{~min}$, and the leakage of calcein was detected using a fluorescence spectrometer. The calcein released from SUVs treated with $0.1 \%$ Triton X-100 and without LfcinB15 treatment was used as a positive and negative control, respectively. As shown in Figure 3, different concentrations of LfcinB15 induced leakage of calcein compared to the controls, suggesting that LfcinB15 can disturb membrane integrity.

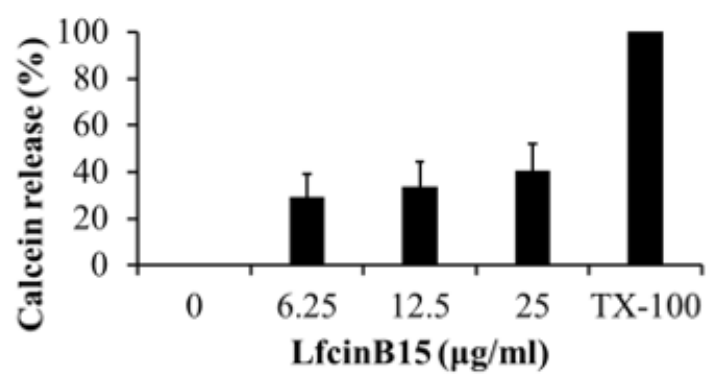

Figure 3. LfcinB15 disturbs the membrane integrity. The release of calcein from SUVs (PC:PE: phosphatidylinositol:ergosterol $=5: 4: 1: 2(w / w / w / w))$ was detected after treatment with different concentrations of LfcinB15 for $5 \mathrm{~min}$. The release of calcein was expressed as a percentage. Calcein release from the SUVs treated with $0.1 \%$ Triton X-100 was defined as $100 \%$ release. TX-100: Triton $X-100$. The error bars represent SD from the mean of three independent experiments. 


\subsection{LfcinB15-Induced ROS Production Is Related to Its Candidacidal Activity}

In addition to disturbance of membrane integrity, the induction of cellular ROS generation is another common mechanism for AMPs against C. albicans [37-39]. Remarkably, an abnormal surface appearance with protuberances was observed in LfcinB15-treated cells (Figure 1d), and this phenotype was correlated with the generation of ROS in C. albicans cells exposed to the antifungal miconazole and other AMPs $[33,40,41]$. Therefore, we further determined whether LfcinB15 can induce ROS generation. Cells were stained with the ROS indicators DHE and $\mathrm{H}_{2}$ DCFDA, and the intracellular ROS level was measured using flow cytometry. As shown in Figure 4a,b, the mean fluorescence intensity (MFI) of cells treated with LfcinB15 was significantly increased compared to that of the untreated control. Moreover, the level of mitochondrial ROS was measured using the mitochondrial superoxide indicator MitoSOX Red. LfcinB15-treated cells had a higher level of MitoSOX Red fluorescence than the control cells (Figure 4c).

(a)

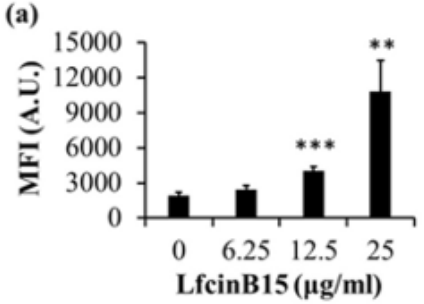

(b)

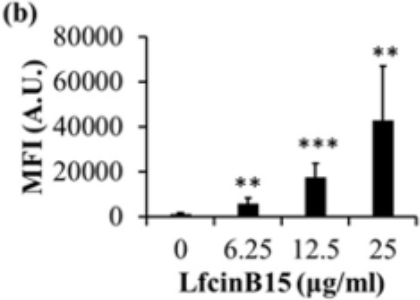

(c)

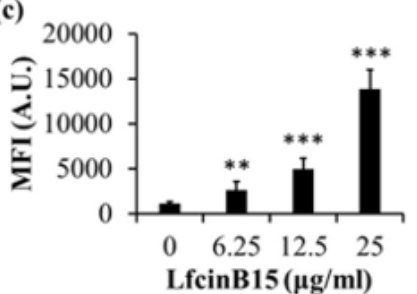

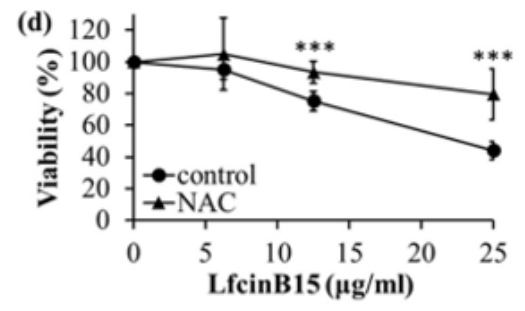

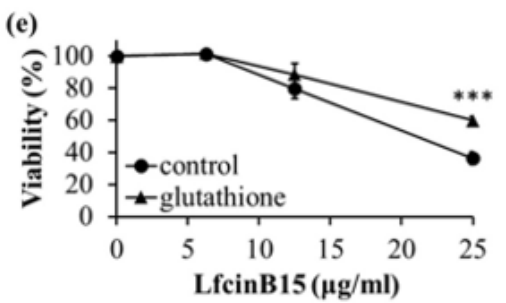

Figure 4. LfcinB15 induces ROS accumulation, and ROS levels correlate with the candidacidal activity of LfcinB15. ROS production was assessed by cell staining with DHE (a), $\mathrm{H}_{2}$ DCFDA (b), and MitoSOX Red (c). The fluorescence intensity of the cells was analyzed using a flow cytometer. MFI, mean fluorescence intensity; A.U., arbitrary units. ${ }^{* *} p<0.01$; ${ }^{* * *} p<0.001$. The effect of ROS scavengers on the candidacidal activity of LfcinB15 was also examined. Cells were pretreated with NAC (d) or glutathione (e), followed by incubation with various concentrations of LfcinB15 as indicated. The data are presented as the mean $\pm \mathrm{SD}$ of three independent experiments. ${ }^{* * *} p<0.001$.

Excessive ROS production leads to oxidative stress, damages vital components of cells and causes cell death [42,43]. Because LfcinB15 induced ROS generation (Figure $4 a-c$ ), the ROS scavengers NAC and glutathione (GSH) were used to correlate ROS with the candidacidal activity of LfcinB15. NAC functions as an antioxidant by increasing the intracellular GSH levels and by directly reacting with ROS for detoxification $[44,45]$. C. albicans cells were pretreated with or without NAC, followed by incubation with various concentrations of LfcinB15, and the number of CFUs was counted. Pretreatment with NAC increased C. albicans viability compared to that of the control without NAC treatment (Figure 4d). This result indicated that NAC can rescue cells from LfcinB15 killing. Furthermore, GSH itself is an important antioxidant, reacting with free thiol groups in biological systems to protect cells from the harmful effects of ROS. Figure 4e shows that LfcinB15-induced cell death can also be rescued in cells pretreated with GSH. Our results suggested that LfcinB15 induced intracellular ROS production, which is likely one of the mechanisms for the candidacidal activity of LfcinB15. 
3.5. The Hog1 and Mkc1 Mitogen-Activated Protein Kinases (MAPKs) Are Activated in Response to $L$ f cinB15

C. albicans Hog1 is a stress-activated kinase that participates in the cell response to osmotic and oxidative stresses [46]. Interestingly, the C. albicans hog $1 \Delta$ mutant is sensitive to the AMPs Hst 5 and human beta-defensins (hBD-2 and hBD-3), suggesting that Hog1 plays a role in the cell response to AMPs $[47,48]$.

Because LfcinB15 induced ROS production that triggered oxidative stress (Figure 4a-e), we also determined the possible involvement of Hog1 in the C. albicans response to LfcinB15. The results showed that the hog1 $1 \Delta$ mutant was more tolerant to LfcinB15 than its wild-type parental strain (Figure 5a). Moreover, the phosphorylation levels of Hog1 were increased, particularly in cells treated with either 12.5 or $25 \mu \mathrm{g} / \mathrm{mL}$ LfcinB15 for 5, 10 and $30 \mathrm{~min}$ (Figure 5b,c). As Hog1 appeared to be involved in the cell response to LfcinB15, two other MAPKs, Mkc1 and Cek1, were also examined. Interestingly, activation of Mkc1, but not Cek1, was also detected (Figure S1 in the Supplementary Materials). Together, LfcinB15 treatment induces Hog1 and Mkc1 phosphorylation, suggesting that these MAPK pathways are involved in cell response against LfcinB15.

(a)

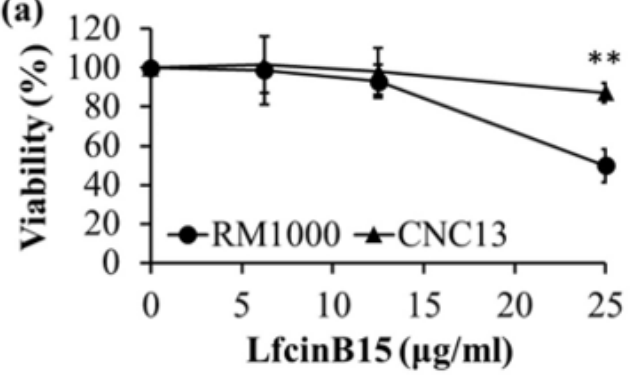

(b)

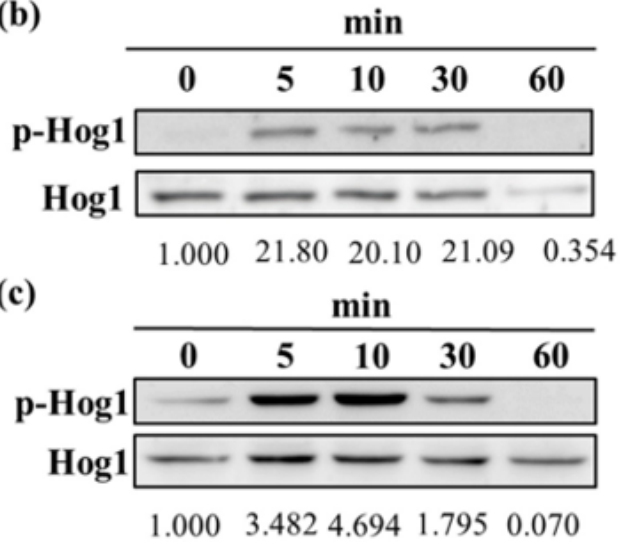

Figure 5. Hog1 is activated in C. albicans treated with LfcinB15. (a) Cells were treated with or without various concentrations of LfcinB, and cell viability is reported as a percentage. RM1000: HOG1/HOG1 [49]; CNC13: $h o g 1 \Delta / h o g 1 \Delta$ [50]. The data are presented as the mean \pm SD of three independent experiments. ${ }^{* *} p<0.01$. Hog1 activation induced by either $12.5 \mu \mathrm{g} / \mathrm{mL}$ (b) or $25 \mu \mathrm{g} / \mathrm{mL}$ (c) LfcinB15 was detected by western blotting and analyzed with ImageJ software. The total Hog1 band of each sample served as the loading control and was used to normalize the phosphor-Hog1 levels indicated by the fold-change values. The data are representative of three independent experiments with identical results.

\subsection{LfcinB15 Also Causes Mitochondrial Dysfunction}

Mitochondria are the major site for ATP synthesis through oxidative phosphorylation that is linked to the electron transport chain (ETC) located in the inner mitochondrial membrane. Additionally, mitochondria are the major source of intracellular ROS that are mainly generated at complexes I and III of the ETC [51]. Because ROS generation is related to the C. albicans- killing activity of LfcinB15 (Figure 4a-e), we further investigated the possible effects of LfcinB15 on mitochondria by measuring the hallmarks of mitochondrial functions, including the mitochondrial membrane potential, the intracellular ATP level and the oxygen consumption rate (OCR) of cells.

The mitochondrial membrane potential $(\Delta \Psi \mathrm{m})$ is an important indicator of mitochondrial activity, and a loss of $\Delta \Psi \mathrm{m}$ represents mitochondrial dysfunction [52]. For the measurement of $\Delta \Psi \mathrm{m}$ changes, two fluorescent dyes, rhodamine 123 and JC-1, were used. C. albicans cells were treated with and without LfcinB15 and stained with rhodamine 123 and JC-1. Simultaneously, cells treated with $\mathrm{H}_{2} \mathrm{O}_{2}$ were used as positive controls. When 
the mitochondrial membrane is depolarized, the fluorescence intensity of rhodamine 123 is expected to be increased, while the wavelength of JC-1 fluorescence will shift from red (maximum at $590 \mathrm{~nm}$ ) to green (maximum at $530 \mathrm{~nm}$ ). As shown in Figure 6a, the MFI of rhodamine 123 was largely increased in cells treated with LfcinB15 or $\mathrm{H}_{2} \mathrm{O}_{2}$ compared to untreated control cells. Moreover, the red-to-green fluorescence ratio of JC-1 was largely decreased in cells treated with LfcinB15 and $\mathrm{H}_{2} \mathrm{O}_{2}$ compared to untreated cells (Figure 6b).
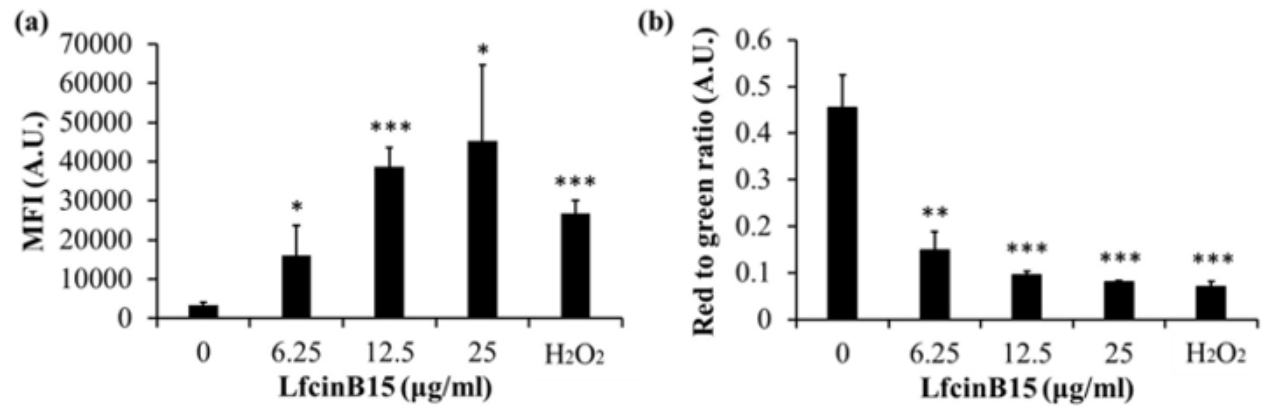

(c)

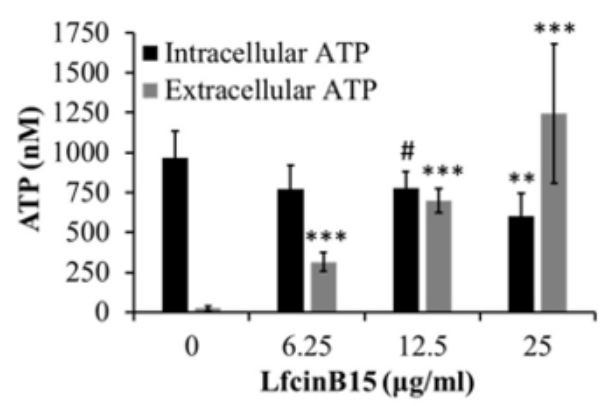

(d)

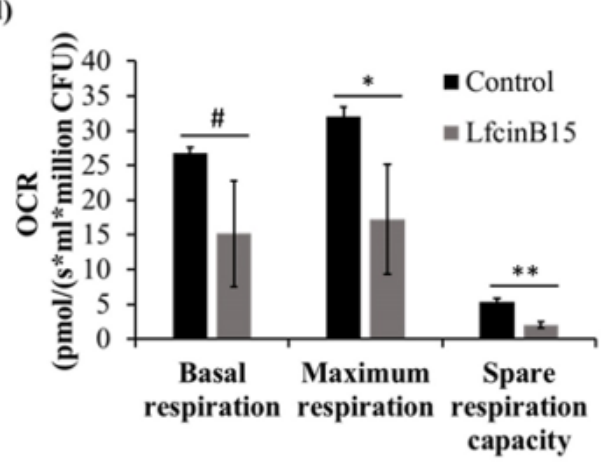

Figure 6. LfcinB15 affects mitochondrial functions. Measurement of mitochondrial membrane potential in cells treated with or without LfcinB15 by rhodamine 123 (a) and JC-1 (b) staining. The results are presented as the mean $\pm \mathrm{SD}$ of three independent experiments. ${ }^{*} p<0.05,{ }^{* *} p<0.01$, *** $p<0.001$. MFI, mean fluorescence intensity; A.U., arbitrary units. (c) The intracellular and extracellular ATP level of each sample was calculated from an ATP standard curve. The results are presented as the mean $\pm \mathrm{SD}$ of at least three independent experiments. $\# p=0.06,{ }^{* *} p<0.01$, *** $p<0.001$. (d) The oxygen consumption rate (OCR) of cells treated with or without a sublethal dose of LfcinB15 $(12.5 \mu \mathrm{g} / \mathrm{mL})$ was measured with high-resolution respirometry using an Oroboros $\mathrm{O} 2 \mathrm{k}$ system. The OCR was defined as pmol oxygen consumed per second per $\mathrm{ml}$ of the sample, and the OCR of each sample was normalized to the number of CFUs. The results are presented as the mean $\pm \mathrm{SD}$ of three independent experiments. $\# p=0.06,{ }^{*} p<0.05,{ }^{* *} p<0.01$.

The intracellular ATP level is tightly controlled to obtain a balance between ATP production and expenditure. Because bioenergetics is a predominant function of mitochondria, a reduction in ATP synthesis is another characteristic of mitochondrial dysfunction. Therefore, C. albicans cells were treated with and without LfcinB15, and the intracellular ATP level was assessed using a luciferin-luciferase assay. As expected, a decrease in the intracellular ATP level was observed in cells treated with various concentrations of LfcinB15 (Figure 6c). However, because LfcinB15 has membrane-disturbing activity (Figure 3), ATP may be leaked out of cells, and this leakage may affect the measurement of the intracellular ATP level. Therefore, in addition to the ATP levels within the cells, the extracellular ATP levels were determined. As shown in Figure $6 c$, the extracellular ATP levels were increased.

In parallel to measuring the mitochondrial membrane potential and ATP synthesis, mitochondrial respiration was also assessed in LfcinB15-exposed cells by measuring the OCR. Cells were treated with or without a sublethal dose of LfcinB15 $(12.5 \mu \mathrm{g} / \mathrm{mL})$ and analyzed with high-resolution respirometry using an Oroboros $\mathrm{O} 2 \mathrm{k}$ system. After measuring the 
basal respiration, CCCP titration was conducted to detect the maximum respiration [53]. The spare respiration capacity was then calculated by subtracting basal respiration from maximal respiration [54]. Figure 6d shows that compared to the untreated control cells, cells treated with LfcinB15 showed a reduced OCR. Moreover, the maximum respiration and spare respiration capacity were reduced, suggesting the ability of LfcinB15 to suppress aerobic respiration. Together, our findings suggested that LfcinB15 treatment leads to mitochondrial dysfunction in C. albicans cells.

\subsection{The Effect of LfcinB15 on Mitochondria Is Associated with the Candidacidal Activity of the Peptide}

To continue to correlate the effect of LfcinB15 on mitochondria in terms of candidacidal activity, various C. albicans mutants that are deficient in mitochondrial ETC and ETC inhibitors were used.

The LfcinB15 susceptibility of five mutants lacking mitochondrial complex I and IV genes (orf19.4758, orf19.7590, mci4, orf19.1710, and cox4) [23] was tested. Orf19.4758 is a putative reductase or dehydrogenase associated with mitochondrial complex I assembly [55], while Orf19.7590 is a putative NADH-ubiquinone oxidoreductase that is one of the mitochondrial complex I core subunits [55]. Moreover, Mci4 is a putative NADH-ubiquinone dehydrogenase and is also related to mitochondrial complex I assembly [55]. Orf19.1710 is also a putative NADH-ubiquinone oxidoreductase related to complex I activity, and Cox4 is a putative cytochrome c oxidase subunit [55]. The results showed that the control SN250 cells were sensitive to LfcinB15, whereas all the mutant strains were resistant to LfcinB15 (Figure 7).
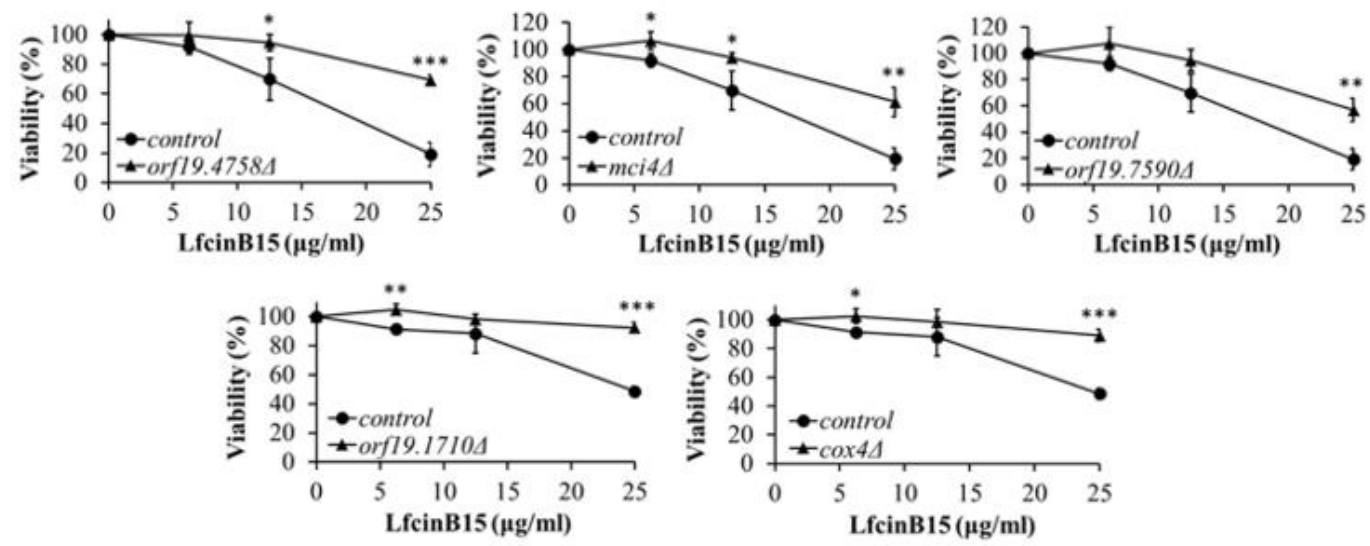

Figure 7. The susceptibility of C. albicans mutants that are deficient in mitochondrial ETC genes to LfcinB15. The viability of C. albicans mutants that are deficient in ETC genes (orf19.4758, mci4, orf19.7590, orf19.1710, and cox4) was compared to that of the parental SN250 strain. The results are presented as the mean \pm SD of three independent experiments. ${ }^{*} p<0.05$, ${ }^{* *} p<0.01,{ }^{* * *} p<0.001$.

Moreover, rotenone is a mitochondrial complex I inhibitor that blocks NADH oxidation, which is the initial step of the ETC. C. albicans cells were treated with rotenone or DMSO (as a negative control), followed by treatment with various concentrations of LfcinB15. As shown in Figure 8a, cells treated with rotenone and LfcinB15 had higher viability than the control cells treated with DMSO and LfcinB15. In addition, $\mathrm{NaN}_{3}$ is an inhibitor of mitochondrial complex IV, and CCCP uncouples the mitochondrial $\triangle \Psi \mathrm{m}$ with the ETC, both inhibiting ATP production. The results showed that cells cotreated with $\mathrm{NaN}_{3}$ and LfcinB15, or with CCCP and LfcinB15, had higher viability than the control cotreated with $\mathrm{NaCl}$ and LfcinB15, or with DMSO and LfcinB15, respectively (Figure 8b,c). 

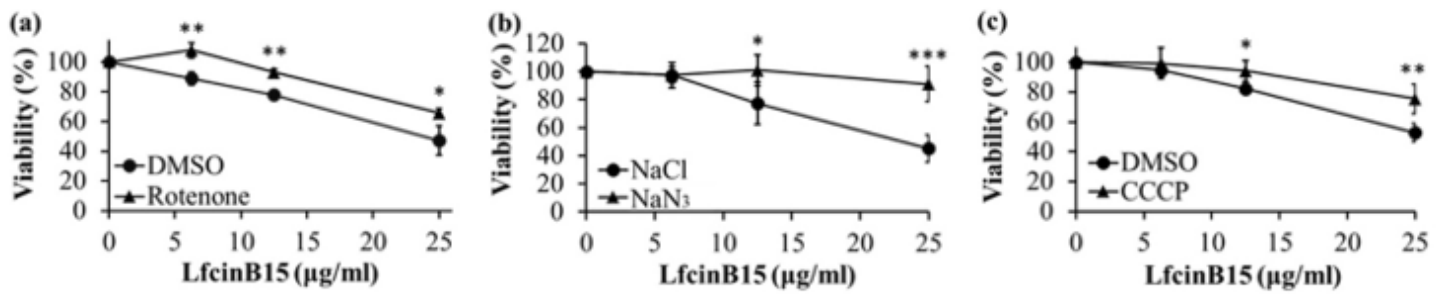

Figure 8. LfcinB15-mediated mitochondrial dysfunction is associated with C. albicans killing. The viability of cells cotreated with LfcinB15 and rotenone (a), $\mathrm{NaN}_{3}$ (b) or CCCP (c) was compared to that of the control as indicated. The results are presented as the mean $\pm \mathrm{SD}$ of three independent experiments. ${ }^{*} p<0.05,{ }^{* *} p<0.01,{ }^{* * *} p<0.001$.

\section{Discussion}

LfcinB15 is an AMP derived from bovine lactoferricin B (LfcinB) and has even higher antibacterial activity than human lactoferricins [18]. However, the antifungal activity and detailed mechanism of LfcinB15 are still mostly unknown. Here, we therefore investigated the mechanisms of action by which LfcinB15 kills C. albicans.

In this study, LfcinB15 exhibited inhibitory activity against planktonic cells, biofilm cells, and clinical isolates of C. albicans and non-albicans Candida species (Figure 1a-c and Table 1). Nevertheless, we also determined the localization of LfcinB15 within C. albicans cells. LfcinB15 is observed not only to associate with the cell surface but also to enter cells and mainly accumulate in vacuoles (Figure 2). Moreover, LfcinB15 exhibits membrane lytic activity (Figure 3). Interestingly, an early study indicated that LfcinB, the parental peptide of LfcinB15, kills C. albicans cells without changing cell wall stability but does induce the release of potassium ions [34]. Hence, similar to that of LfcinB, our findings suggest that disturbing membrane integrity is one of the mechanisms related to the inhibitory activity of LfcinB15.

Redox homeostasis is vital for C. albicans survival. The impacts of AMPs on mitochondrial redox balance to induce ROS accumulation have been reported in several studies [38,56,57]. Increased ROS levels lead to oxidative stress, and ROS can induce cell death. In this study, LfcinB15 induced ROS generation that may cause oxidative stress, and ROS accumulation was associated with the killing activity of LfcinB15 (Figures 1d and $4 \mathrm{a}-\mathrm{e})$. Furthermore, mitochondria are considered a major source of ROS [42], and mitochondrial dysfunction is often associated with the accumulation of ROS [58]. Indeed, several antifungal agents and AMPs that induce ROS generation can also cause mitochondrial dysfunction. For example, both kalopanaxsaponin A (KPA) and the AMP hepcidin 25 induce ROS generation in C. albicans [56,59]. Additionally, KPA causes a significant decrease in the intracellular ATP level in a dose-dependent manner [59], and hepcidin 25 is able to reduce the mitochondrial membrane potential [56]. Because LfcinB15 induces ROS generation, several hallmarks of mitochondrial dysfunction were thus examined. In Figure 6a-d, depolarization of the mitochondrial membrane, decreased intracellular ATP content and reduced OCR are shown in cells treated with LfcinB15. Mitochondrial ROS generation mainly occurred through the production of superoxide from complexes I and III of the ETC, although other sites can also contribute. Importantly, mitochondrial dysfunction appears to be associated with the C. albicans-killing activity of LfcinB15, as further indicated by different ETC inhibitors including rotenone, NaN3 and CCCP, and mitochondrial-deficient mutants (Figure 7). Furthermore, it is interesting to note that cells cotreated with the ETC inhibitors and LfcinB15 had higher viability than the controls (Figure $8 \mathrm{a}-\mathrm{c}$ ). Because ETC generates a proton gradient that drives ATP synthesis (in complex V) and ROS generation occurs in the ETC, all the inhibitors blocking ETC can lead to reduced ROS generation and ATP synthesis. Interestingly, Helmerhorst et al. also demonstrated that cells cotreated with $\mathrm{NaN}_{3}$ or cyanide can rescue the killing by Hst 5 , and concluded that Hst 5 targets the energized mitochondria [60]. Together, our results suggest that ROS, oxidative stress and mitochondrial dysfunction are also related to $C$. albicans killing by LfcinB15, apart from membrane disturbance. 
In addition to the change of intracellular ATP levels, LfcinB15 is able to induce the release of ATP into the extracellular environment (Figure 6c). Interestingly, released cellular ATP is associated with candidacidal activity by some cationic AMPs and regulation of cellular functions in higher eukaryotes [11,61]. For example, the addition of apyrase, an ATP scavenger, can reduce Hst 5-mediated killing, suggesting a direct connection between released ATP and C. albicans cell death [62]. Moreover, released ATP modulates functions of immune cells through its activation of purinergic receptors [63]. Although the underlying mechanisms are not fully understood, treatment with purinergic antagonists prevents Hst 5-mediated C. albicans killing [64]. Finally, the release of ATP induced by Hst 5 occurs without membrane lysis and is mediated by ion transporters [61]. However, our results indicated that LfcinB15 is able to disturb membrane integrity and also induce ATP release (Figure 6c). Therefore, several questions still need to be addressed to discern different mechanisms between LfcinB15 and Hst 5. For example, how does LfcinB15 interact with C. albicans cells? Is recognition and binding with cell surface proteins or other components required for the candidacidal activity of LfcinB15? Finally, is released ATP involved in LfcinB15-mediated cell death?

Moreover, LfcinB15 treatment induced Hog1 activation, but unexpectedly, the hog1 $\Delta$ mutant was more resistant to LfcinB15 (Figure 5a-c). Interestingly, similar findings have been reported for other fungal pathogens treated with different antifungal reagents. For example, the OSC1 gene encodes an ortholog of Hog1 in the plant pathogen Colletorichum lagenarium. Treatment with fludioxonil activates Osc1 phosphorylation, whereas the osc1 $\Delta$ mutant was more resistant to fludioxonil [65]. Moreover, C. albicans Nik1 is a two-component histidine kinase functioning upstream of Hog1 [66-68]. Using a Saccharomyces cerevisiae strain (named CaNik1) with heterologous expression of C. albicans Nik1, Hog1 is activated upon treatment with fludioxonil [66]. The CaNik1 strain with HOG1-deletion is also resistant to fludioxonil [66]. Finally, other than Hog1, LfcinB15 can also induce the activation of Mkc1 (Figure S1 in the Supplementary Materials). Although the LfcinB15 resistance of the hog1 $1 \Delta$ mutant and possible interplay between Hog1 and Mkc1 signaling pathways remain elusive, our study shows the complexity in cell responses to AMPs.

Of special interest in the present study is that LfcinB15 intracellularly accumulates in vacuoles, whereas mitochondrial functions are affected. Notably, similar phenomena were previously reported from studies of Hst 5 [11,57]. These observations raise an interesting question regarding how LfcinB15 functions between mitochondria and vacuoles. Recently, a molecular hub connecting the mitochondria and vacuole was identified in baker's yeast $S$. cerevisiae $[69,70]$. Specifically, this hub is called the vacuole and mitochondria patch (vCLAMP) and consists of several proteins including Mcp1, Vam6, and the small GTPase Ypt7 [71]. However, there is only very limited information about the vCLAMP in C. albicans. Vam6 is involved in the maintenance of mitochondrial and vacuolar functions under oxidative stress in C. albicans [72]. In addition, Mcp1 contributes not only to mitochondrial maintenance, but also to mitophagy [73]. Because LfcinB15 treatment is likely to induce oxidative stress and causes mitochondrial dysfunction, it is possible that LfcinB15 killing is associated with the vCLAMP. Ypt72 is the C. albicans homolog of S. cerevisiae Ypt7 (http: / / www.candidagenome.org, accessed on 17 May 2021). Intriguingly, we found that the $C$. albicans ypt72 $\Delta$ mutant was more tolerant to LfcinB15 than its wild-type parental strain (Figure S2 in the Supplementary Materials). However, the details underlying this finding for the possible relationship between LfcinB15 and the vCLAMP must be further investigated.

Considering the potential future use of LfcinB15, we have also initiated an investigation to determine the efficacy of LfcinB15 against different Candida species. LfcinB15 is a broad-spectrum AMP that deploys its activity against different clinical isolates of Candida species (Table 1). Importantly, many of these isolates are fluconazole resistant. Moreover, two C. glabrata isolates were much more resistant to LfcinB15 than C. albicans and other tested isolates. Similarly, resistance of C. glabrata to other AMPs such as $p-113$, VLL-28 and some other LfcinB-derived peptides was also reported [33-35]. In terms of 
mitochondrial structure and function, several possible reasons may be used to explain the different LfcinB15 susceptibility between C. glabrata and C. albicans. The mitochondrial ETC of $C$. glabrata resembles that of $S$. cerevisiae rather than C. albicans, and does not express a multi-subunit complex I [74]. In addition, the OCR of C. glabrata cells is significantly lower than that of C. albicans [75]. However, clarifying the different LfcinB15 susceptibility will require more clinical isolates of C. glabrata for future study.

\section{Conclusions}

In summary, we demonstrated that LfcinB15 exhibits anti-Candida activity against planktonic cells, biofilm cells and clinical isolates. Moreover, LfcinB15 possesses multiple modes of action in C. albicans killing, including causing mitochondrial damage. Recently, much attention has been paid to exploring mitochondria as new targets for the development of antifungals [76,77]. Here, our findings of LfcinB15 imply that communication between organelles may also be involved in AMP-mediated killing, although the detailed mechanisms remain to be elucidated. Overall, our results, in combination with others, highlight the complex mechanisms that may be used by AMPs and suggest the potential use of AMPs in therapeutic applications for infectious diseases.

Supplementary Materials: The following are available online at https: / www.mdpi.com/article/ 10.3390/jof7070519/s1. Figure S1: LfcinB15 treatment activates C. albicans Mkc1. Figure S2: The C. albicans ypt72-deleted mutant is more tolerant to LfcinB15.

Author Contributions: Conceptualization, C.-K.C., M.-C.K. and C.-Y.L.; methodology, C.-K.C., M.C.K. and C.-Y.L.; validation, C.-K.C. and C.-Y.L.; investigation, C.-K.C.; resources, C.-Y.L.; data curation, C.-K.C. and C.-Y.L.; writing-original draft preparation, C.-K.C. and C.-Y.L.; writingreview and editing, C.-K.C., M.-C.K. and C.-Y.L.; visualization, C.-K.C. and C.-Y.L.; supervision, C.-Y.L.; project administration, C.-Y.L.; funding acquisition, M.-C.K. and C.-Y.L. All authors have read and agreed to the published version of the manuscript.

Funding: This work was supported by the Ministry of Science and Technology (MOST), Taiwan (grants MOST109-2311-B-007-001-MY3 and MOST108-2311-B-007-005 to CYL and grant MOST1072313-B-007-002-MY to MKC).

Institutional Review Board Statement: Not applicable.

Informed Consent Statement: Not applicable.

Data Availability Statement: The data presented in this study are available within the article and the Supplementary Materials.

Acknowledgments: We thank Hsiu-Jung Lo for generously providing the clinical strains and support from the confocal imaging core at National Tsing Hua University (sponsored by MOST108-2731-M007-001).

Conflicts of Interest: The authors declare no conflict of interest. The funders had no role in the design of the study; in the collection, analyses, or interpretation of data; in the writing of the manuscript, or in the decision to publish the results.

\section{References}

1. Sardi, J.C.; Scorzoni, L.; Bernardi, T.; Fusco-Almeida, A.M.; Mendes Giannini, M.J. Candida species: Current epidemiology, pathogenicity, biofilm formation, natural antifungal products and new therapeutic options. J. Med. Microbiol. 2013, 62, 10-24. [CrossRef] [PubMed]

2. Ben-Ami, R. Treatment of Invasive Candidiasis: A Narrative Review. J. Fungi 2018, 4, 97. [CrossRef] [PubMed]

3. Brissaud, O.; Guichoux, J.; Harambat, J.; Tandonnet, O.; Zaoutis, T. Invasive fungal disease in PICU: Epidemiology and risk factors. Ann. Intensive Care 2012, 2, 6. [CrossRef] [PubMed]

4. Arastehfar, A.; Lass-Florl, C.; Garcia-Rubio, R.; Daneshnia, F.; Ilkit, M.; Boekhout, T.; Gabaldon, T.; Perlin, D.S. The quiet and underappreciated rise of drug-resistant invasive fungal pathogens. J. Fungi 2020, 6, 138. [CrossRef] [PubMed]

5. Bondaryk, M.; Staniszewska, M.; Zielinska, P.; Urbanczyk-Lipkowska, Z. Natural antimicrobial peptides as inspiration for design of a new generation antifungal compounds. J. Fungi 2017, 3, 46. [CrossRef] 
6. Mahlapuu, M.; Hakansson, J.; Ringstad, L.; Bjorn, C. Antimicrobial peptides: An emerging category of therapeutic agents. Front. Cell. Infect. Microbiol. 2016, 6, 194. [CrossRef] [PubMed]

7. Steckbeck, J.D.; Deslouches, B.; Montelaro, R.C. Antimicrobial peptides: New drugs for bad bugs? Expert Opin. Biol. Ther. 2014, 14, 11-14. [CrossRef]

8. Midura-Nowaczek, K.; Markowska, A. Antimicrobial peptides and their analogs: Searching for new potential therapeutics. Perspect. Med. Chem. 2014, 6, S13215. [CrossRef]

9. Hollmann, A.; Martinez, M.; Maturana, P.; Semorile, L.C.; Maffia, P.C. Antimicrobial peptides: Interaction with model and biological membranes and synergism with chemical antibiotics. Front. Chem. 2018, 6, 204. [CrossRef]

10. Le, C.F.; Fang, C.M.; Sekaran, S.D. Intracellular targeting mechanisms by antimicrobial peptides. Antimicrob. Agents Chemother. 2017, 61, e02340-16. [CrossRef]

11. Puri, S.; Edgerton, M. How does it kill?: Understanding the candidacidal mechanism of salivary histatin 5. Eukaryot. Cell 2014, 13, 958-964. [CrossRef]

12. Mardirossian, M.; Perebaskine, N.; Benincasa, M.; Gambato, S.; Hofmann, S.; Huter, P.; Muller, C.; Hilpert, K.; Innis, C.A.; Tossi, A.; et al. The dolphin proline-rich antimicrobial peptide Tur1A inhibits protein synthesis by targeting the bacterial ribosome. Cell Chem. Biol. 2018, 25, 530-539.e7. [CrossRef] [PubMed]

13. Adlerova, L.; Bartoskova, A.; Faldyna, M. Lactoferrin: A review. Vet. Med. 2008, 53, 457-468. [CrossRef]

14. Rosa, L.; Cutone, A.; Lepanto, M.S.; Paesano, R.; Valenti, P. Lactoferrin: A natural glycoprotein involved in iron and inflammatory homeostasis. Int. J. Mol. Sci. 2017, 18, 1985. [CrossRef]

15. Jenssen, H.; Hancock, R.E. Antimicrobial properties of lactoferrin. Biochimie 2009, 91, 19-29. [CrossRef]

16. Gifford, J.L.; Hunter, H.N.; Vogel, H.J. Lactoferricin: A lactoferrin-derived peptide with antimicrobial, antiviral, antitumor and immunological properties. Cell. Mol. Life Sci. 2005, 62, 2588-2598. [CrossRef] [PubMed]

17. Bellamy, W.; Takase, M.; Yamauchi, K.; Wakabayashi, H.; Kawase, K.; Tomita, M. Identification of the bactericidal domain of lactoferrin. Biochim. Biophys. Acta BBA Protein Struct. Mol. Enzymol. 1992, 1121, 130-136. [CrossRef]

18. Vorland, L.H.; Ulvatne, H.; Andersen, J.; Haukland, H.; Rekdal, O.; Svendsen, J.S.; Gutteberg, T.J. Lactoferricin of bovine origin is more active than lactoferricins of human, murine and caprine origin. Scand. J. Infect. Dis. 1998, 30, 513-517.

19. Bruni, N.; Capucchio, M.T.; Biasibetti, E.; Pessione, E.; Cirrincione, S.; Giraudo, L.; Corona, A.; Dosio, F. Antimicrobial activity of lactoferrin-related peptides and applications in human and veterinary medicine. Molecules 2016, 21, 752. [CrossRef]

20. Hao, Y.; Yang, N.; Teng, D.; Wang, X.; Mao, R.; Wang, J. A review of the design and modification of lactoferricins and their derivatives. Biometals 2018, 31, 331-341. [CrossRef]

21. Fernandes, K.E.; Carter, D.A. The antifungal activity of lactoferrin and its derived peptides: Mechanisms of action and synergy with drugs against fungal pathogens. Front. Microbiol. 2017, 8, 2. [CrossRef] [PubMed]

22. Liu, Y.; Han, F.; Xie, Y.; Wang, Y. Comparative antimicrobial activity and mechanism of action of bovine lactoferricin-derived synthetic peptides. Biometals 2011, 24, 1069-1078. [CrossRef] [PubMed]

23. Noble, S.M.; French, S.; Kohn, L.A.; Chen, V.; Johnson, A.D. Systematic screens of a Candida albicans homozygous deletion library decouple morphogenetic switching and pathogenicity. Nat. Genet. 2010, 42, 590-598. [CrossRef]

24. Yu, H.Y.; Tu, C.H.; Yip, B.S.; Chen, H.L.; Cheng, H.T.; Huang, K.C.; Lo, H.J.; Cheng, J.W. Easy strategy to increase salt resistance of antimicrobial peptides. Antimicrob. Agents Chemother. 2011, 55, 4918-4921. [CrossRef]

25. Wayne, P.A. Reference Method for Broth Dilution Antifungal Susceptibility Testing of Yeasts, 3rd ed.; Approved Standard, CLSI Document M27-A3; Clinical and Laboratory Standards Institute: Annapolis Junction, MD, USA, 2008.

26. Rothstein, D.M.; Spacciapoli, P.; Tran, L.T.; Xu, T.; Roberts, F.D.; Dalla Serra, M.; Buxton, D.K.; Oppenheim, F.G.; Friden, P. Anticandida activity is retained in P-113, a 12-amino-acid fragment of histatin 5. Antimicrob. Agents Chemother. 2001, 45, 1367-1373. [CrossRef]

27. Chen, H.F.; Lan, C.Y. Role of SFP1 in the regulation of Candida albicans biofilm formation. PLoS ONE 2015, 10, e0129903. [CrossRef] [PubMed]

28. Huang, W.N.; Sue, S.C.; Wang, D.S.; Wu, P.L.; Wu, W.G. Peripheral binding mode and penetration depth of cobra cardiotoxin on phospholipid membranes as studied by a combined FTIR and computer simulation approach. Biochemistry 2003, 42, 7457-7466. [CrossRef]

29. Xue, Y.P.; Kao, M.C.; Lan, C.Y. Novel mitochondrial complex I-inhibiting peptides restrain NADH dehydrogenase activity. Sci. Rep. 2019, 9, 13694. [CrossRef]

30. Chen, Y.W.; Yeh, Y.C.; Chen, H.F.; Chen, R.C.; Lin, G.Y.; Chen, Y.T.; Lan, C.Y. The small GTPase Rhb1 is involved in the cell response to fluconazole in Candida albicans. FEMS Yeast Res. 2019, 19, foz005. [CrossRef]

31. Lis, M.; Bobek, L.A. Proteomic and metabolic characterization of a Candida albicans mutant resistant to the antimicrobial peptide MUC7 12-mer. FEMS Immunol. Med. Microbiol. 2008, 54, 80-91. [CrossRef]

32. Wall, G.; Montelongo-Jauregui, D.; Vidal Bonifacio, B.; Lopez-Ribot, J.L.; Uppuluri, P. Candida albicans biofilm growth and dispersal: Contributions to pathogenesis. Curr. Opin. Microbiol. 2019, 52, 1-6. [CrossRef]

33. Lin, G.Y.; Chen, H.F.; Xue, Y.P.; Yeh, Y.C.; Chen, C.L.; Liu, M.S.; Cheng, W.C.; Lan, C.Y. The antimicrobial peptides P-113Du and P-113Tri function against Candida albicans. Antimicrob. Agents Chemother. 2016, 60, 6369-6373. [CrossRef]

34. Wakabayashi, H.; Hiratani, T.; Uchida, K.; Yamaguchi, H. Antifungal spectrum and fungicidal mechanism of an N-terminal peptide of bovine lactoferrin. J. Infect. Chemother. 1996, 1, 185-189. [CrossRef] 
35. Roscetto, E.; Contursi, P.; Vollaro, A.; Fusco, S.; Notomista, E.; Catania, M.R. Antifungal and anti-biofilm activity of the first cryptic antimicrobial peptide from an archaeal protein against Candida spp. clinical isolates. Sci. Rep. 2018, 8, 17570. [CrossRef]

36. Kumar, P.; Kizhakkedathu, J.N.; Straus, S.K. Antimicrobial peptides: Diversity, mechanism of action and strategies to improve the activity and biocompatibility in vivo. Biomolecules 2018, 8, 4. [CrossRef] [PubMed]

37. Kim, S.; Lee, D.G. Role of calcium in reactive oxygen species-induced apoptosis in Candida albicans: An antifungal mechanism of antimicrobial peptide, PMAP-23. Free Radic. Res. 2019, 53, 8-17. [CrossRef]

38. Seyedjavadi, S.S.; Khani, S.; Eslamifar, A.; Ajdary, S.; Goudarzi, M.; Halabian, R.; Akbari, R.; Zare-Zardini, H.; Imani Fooladi, A.A.; Amani, J.; et al. The antifungal peptide MCh-AMP1 derived from Matricaria chamomilla inhibits Candida albicans growth via inducing ROS generation and altering fungal cell membrane permeability. Front. Microbiol. 2019, 10, 3150. [CrossRef] [PubMed]

39. Wang, K.; Dang, W.; Xie, J.; Zhu, R.; Sun, M.; Jia, F.; Zhao, Y.; An, X.; Qiu, S.; Li, X.; et al. Antimicrobial peptide protonectin disturbs the membrane integrity and induces ROS production in yeast cells. Biochim. Biophys. Acta 2015, 1848, $2365-2373$. [CrossRef]

40. De Nollin, S.; Borgers, M. Scanning electron microscopy of Candida albicans after in vitro treatment with miconazole. Antimicrob. Agents Chemother. 1975, 7, 704-711. [CrossRef] [PubMed]

41. Vandenbosch, D.; Braeckmans, K.; Nelis, H.J.; Coenye, T. Fungicidal activity of miconazole against Candida spp. biofilms. J. Antimicrob. Chemother. 2010, 65, 694-700. [CrossRef]

42. Perrone, G.G.; Tan, S.X.; Dawes, I.W. Reactive oxygen species and yeast apoptosis. Biochim. Biophys. Acta 2008, 1783, 1354-1368. [CrossRef]

43. Farrugia, G.; Balzan, R. Oxidative stress and programmed cell death in yeast. Front. Oncol. 2012, 2, 64. [CrossRef] [PubMed]

44. Lee, W.; Lee, D.G. Reactive oxygen species modulate itraconazole-induced apoptosis via mitochondrial disruption in Candida albicans. Free Radic. Res. 2018, 52, 39-50. [CrossRef] [PubMed]

45. Sun, S.Y. N-acetylcysteine, reactive oxygen species and beyond. Cancer Biol. Ther. 2010, 9, 109-110. [CrossRef]

46. Day, A.M.; Quinn, J. Stress-activated protein kinases in human fungal pathogens. Front. Cell Infect. Microbiol. $2019,9,261$. [CrossRef]

47. Argimon, S.; Fanning, S.; Blankenship, J.R.; Mitchell, A.P. Interaction between the Candida albicans high-osmolarity glycerol (HOG) pathway and the response to human beta-defensins 2 and 3. Eukaryot. Cell 2011, 10, 272-275. [CrossRef]

48. Vylkova, S.; Jang, W.S.; Li, W.; Nayyar, N.; Edgerton, M. Histatin 5 initiates osmotic stress response in Candida albicans via activation of the Hog1 mitogen-activated protein kinase pathway. Eukaryot. Cell 2007, 6, 1876-1888. [CrossRef]

49. Negredo, A.; Monteoliva, L.; Gil, C.; Pla, J.; Nombela, C. Cloning, analysis and one-step disruption of the ARG5, 6 gene of Candida albicans. Microbiology 1997, 143, 297-302. [CrossRef] [PubMed]

50. Alonso-Monge, R.; Navarro-Garcia, F.; Molero, G.; Diez-Orejas, R.; Gustin, M.; Pla, J.; Sanchez, M.; Nombela, C. Role of the mitogen-activated protein kinase Hog1p in morphogenesis and virulence of Candida albicans. J. Bacteriol. 1999, 181, 3058-3068. [CrossRef]

51. Zhao, R.Z.; Jiang, S.; Zhang, L.; Yu, Z.B. Mitochondrial electron transport chain, ROS generation and uncoupling. Int. J. Mol. Med. 2019, 44, 3-15. [CrossRef] [PubMed]

52. Zorova, L.D.; Popkov, V.A.; Plotnikov, E.Y.; Silachev, D.N.; Pevzner, I.B.; Jankauskas, S.S.; Babenko, V.A.; Zorov, S.D.; Balakireva, A.V.; Juhaszova, M.; et al. Mitochondrial membrane potential. Anal. Biochem. 2018, 552, 50-59. [CrossRef]

53. Duvenage, L.; Munro, C.; Gourlay, C. High resolution respirometry in Candida albicans. Bio Protocol 2019, 9, e3361. [CrossRef]

54. Brand, M.D.; Nicholls, D.G. Assessing mitochondrial dysfunction in cells. Biochem. J. 2011, 435, 297-312. [CrossRef]

55. Skrzypek, M.S.; Binkley, J.; Binkley, G.; Miyasato, S.R.; Simison, M.; Sherlock, G. The Candida genome database (CGD): Incorporation of Assembly 22, systematic identifiers and visualization of high throughput sequencing data. Nucleic Acids Res. 2017, 45, D592-D596. [CrossRef]

56. Chen, R.C.; Lan, C.Y. Human antimicrobial peptide hepcidin 25-induced apoptosis in Candida albicans. Microorganisms 2020, 8, 585. [CrossRef]

57. Helmerhorst, E.J.; Troxler, R.F.; Oppenheim, F.G. The human salivary peptide histatin 5 exerts its antifungal activity through the formation of reactive oxygen species. Proc. Natl. Acad. Sci. USA 2001, 98, 14637-14642. [CrossRef] [PubMed]

58. Murphy, M.P. Mitochondrial dysfunction indirectly elevates ROS production by the endoplasmic reticulum. Cell Metab. 2013, 18, 145-146. [CrossRef]

59. Li, Y.; Shan, M.; Zhu, Y.; Yao, H.; Li, H.; Gu, B.; Zhu, Z. Kalopanaxsaponin A induces reactive oxygen species mediated mitochondrial dysfunction and cell membrane destruction in Candida albicans. PLoS ONE 2020, 15, e0243066. [CrossRef]

60. Helmerhorst, E.J.; Breeuwer, P.; van't Hof, W.; Walgreen-Weterings, E.; Oomen, L.C.; Veerman, E.C.; Amerongen, A.V.; Abee, T. The cellular target of histatin 5 on Candida albicans is the energized mitochondrion. J. Biol. Chem. 1999, 274, 7286-7291. [CrossRef]

61. Vylkova, S.; Sun, J.N.; Edgerton, M. The role of released ATP in killing Candida albicans and other extracellular microbial pathogens by cationic peptides. Purinergic Signal. 2007, 3, 91-97. [CrossRef]

62. Koshlukova, S.E.; Araujo, M.W.; Baev, D.; Edgerton, M. Released ATP is an extracellular cytotoxic mediator in salivary histatin 5-induced killing of Candida albicans. Infect. Immun. 2000, 68, 6848-6856. [CrossRef] [PubMed]

63. Ledderose, C.; Junger, W.G. Mitochondria synergize with P2 receptors to regulate human T cell function. Front. Immunol. 2020, 11, 549889. [CrossRef] 
64. Koshlukova, S.E.; Lloyd, T.L.; Araujo, M.W.; Edgerton, M. Salivary histatin 5 induces non-lytic release of ATP from Candida albicans leading to cell death. J. Biol. Chem. 1999, 274, 18872-18879. [CrossRef]

65. Kojima, K.; Takano, Y.; Yoshimi, A.; Tanaka, C.; Kikuchi, T.; Okuno, T. Fungicide activity through activation of a fungal signalling pathway. Mol. Microbiol. 2004, 53, 1785-1796. [CrossRef] [PubMed]

66. Buschart, A.; Gremmer, K.; El-Mowafy, M.; van den Heuvel, J.; Mueller, P.P.; Bilitewski, U. A novel functional assay for fungal histidine kinases group III reveals the role of HAMP domains for fungicide sensitivity. J. Biotechnol. 2012, 157, 268-277. [CrossRef]

67. Cui, S.; Hassan, R.Y.; Heintz-Buschart, A.; Bilitewski, U. Regulation of Candida albicans interaction with macrophages through the activation of HOG pathway by genistein. Molecules 2016, 21, 162. [CrossRef] [PubMed]

68. El-Mowafy, M.; Bahgat, M.M.; Bilitewski, U. Deletion of the HAMP domains from the histidine kinase CaNik1p of Candida albicans or treatment with fungicides activates the MAP kinase Hog1p in S. cerevisiae transformants. BMC Microbiol. 2013, 13, 209. [CrossRef]

69. Elbaz-Alon, Y.; Rosenfeld-Gur, E.; Shinder, V.; Futerman, A.H.; Geiger, T.; Schuldiner, M. A dynamic interface between vacuoles and mitochondria in yeast. Dev. Cell 2014, 30, 95-102. [CrossRef]

70. Honscher, C.; Mari, M.; Auffarth, K.; Bohnert, M.; Griffith, J.; Geerts, W.; van der Laan, M.; Cabrera, M.; Reggiori, F.; Ungermann, C. Cellular metabolism regulates contact sites between vacuoles and mitochondria. Dev. Cell 2014, 30, 86-94. [CrossRef]

71. Soto-Heredero, G.; Baixauli, F.; Mittelbrunn, M. Interorganelle communication between mitochondria and the endolysosomal system. Front. Cell Dev. Biol. 2017, 5, 95. [CrossRef]

72. Mao, X.; Yang, L.; Liu, Y.; Ma, C.; Ma, T.; Yu, Q.; Li, M. Vacuole and mitochondria patch (vCLAMP) protein Vam6 is involved in maintenance of mitochondrial and vacuolar functions under oxidative stress in Candida albicans. Antioxidants 2021, 10, 136. [CrossRef]

73. Mao, X.; Yang, L.; Fan, Y.; Wang, J.; Cui, D.; Yu, D.; Yu, Q.; Li, M. The vacuole and mitochondria patch (vCLAMP) protein Mcp1 is involved in maintenance of mitochondrial function and mitophagy in Candida albicans. Front. Microbiol. 2021, 12, 633380. [CrossRef]

74. Duvenage, L.; Munro, C.A.; Gourlay, C.W. The potential of respiration inhibition as a new approach to combat human fungal pathogens. Curr. Genet. 2019, 65, 1347-1353. [CrossRef] [PubMed]

75. Duvenage, L.; Pentland, D.R.; Munro, C.A.; Gourlay, C.W. An analysis of respiratory function and mitochondrial morphology in Candida albicans. bioRxiv 2019, 697516. [CrossRef]

76. Li, D.; Calderone, R. Exploiting mitochondria as targets for the development of new antifungals. Virulence 2017, 8, 159-168. [CrossRef] [PubMed]

77. Sun, N.; Parrish, R.S.; Calderone, R.A.; Fonzi, W.A. Unique, diverged, and conserved mitochondrial functions influencing Candida albicans respiration. mBio 2019, 10, e00300-19. [CrossRef] 